\title{
Asymptotic Behavior of a Bi-Dimensional Hybrid System
}

\author{
Pedro Gamboa', Jaime E. Muñoz ${ }^{1,2}$, Octavio Vera ${ }^{3}$, Margareth Alves ${ }^{4}$ \\ ${ }^{1}$ Departamento de Matemática, Instituto de Matemáticas, Universidade Federal do Rio de Janeiro, \\ Rio de Janeiro, Brazil \\ ${ }^{2}$ Laboratorio Nacional de Computação Científica, Petrópolis, Brazil \\ ${ }^{3}$ Departamento de Matemática, Facultad de Ciencias, Universidad del Bo-Bo, Concepción, Chile \\ ${ }^{4}$ Departamento de Matemática, UFV, Viçosa, Brazil \\ Email: pgamboa@im.ufri.br, rivera@im.ufri.br, rivera@Incc.br, overa@ubiobio.cl, malves@ufv.br
}

Received 3 June 2015; accepted 10 July 2015; published 14 July 2015

Copyright (C) 2015 by authors and Scientific Research Publishing Inc.

This work is licensed under the Creative Commons Attribution International License (CC BY).

http://creativecommons.org/licenses/by/4.0/

(c) () Open Access

\author{
Abstract \\ We study the asymptotic behavior of the solutions of a Hybrid System wrapping an elliptic opera- \\ tor. \\ Keywords \\ Hybrid System, Compressible, Stabilization, Asymptotic Behavior, Decay Rate, Generator \\ Infinitesimal, Polynomial Decay
}

\section{Introduction}

In this paper, we address some issues related to the asymptotic behavior a hybrid system with two types of vibrations of different nature. The model under consideration is inspired in and introduced in [1]. However, there are some important differences between these two models. In [1] the flexible part of the boundary $\Gamma_{0}$ is occupied by a flexible damped beam instead of a flexible. Most of the relevant properties see [2]. In [3] the authors are interested on the existence of periodic solutions of this system. Due to the localization of the damping term in a relatively small part of the boundary and to the effect of the hybrid structure of the system, the existence of periodic solutions holds for a restricted class of non homogeneous terms. Some resonance-type phenomena are also exhibited. Cindea, Sorin and Pazoto [4] consider the motion of a stretched string coupled with a rigid body at one end and we study the existence of periodic solution when a periodic force facts on the body. The main difficulty of the study is related to the weak dissipation that characterizes this hybrid system, which does not ensure a uniform decay rate of the energy. For more examples of hybrid systems see [5] [6]. We refer to [7] for a 
discussion on the model and references therein. In [8] the authors to discern exact controllability properties of two coupled wave equations, one of which holds on the interior of a bounded open domain $\Omega$, and the other on a segment $\Gamma_{0}$ of the boundary $\partial \Omega$. Moreover, the coupling is accomplished through terms on the boundary. Because of the particular physical application involved the attenuation of acoustic waves within a chamber by means of active controllers on the chamber walls control is to be implemented on the boundary only.

We consider the bi-dimensional cavity $\Omega=\Omega_{1} \backslash \bar{\Omega}_{0}$ and that $\Omega_{0}$ an open class $C^{2}$ with limited boundary contained in $\Omega_{1}$, filled with an elastic, in viscid, compressible fluid, in which the acoustic vibrations are coupled with the mechanical vibration of a string located in the subset $\Gamma_{0}=\{(x, 0) ; x \in(0,1)\}$ a part of the boundary of omega of $\Omega_{1}, \Gamma_{1}=\partial \Omega_{1} \backslash \Gamma_{0}$ and $\Gamma=\partial \Omega_{1} \cup \partial \Omega_{0}$ with $\partial \Omega_{1} \cap \partial \Omega_{0}=\phi$, is boundary of $\Omega$. The subset $\Gamma_{1}$ is assumed to be rigid and we impose zero normal velocity of the fluids on it. The subset $\Gamma_{0}$ is supposed to be flexible and occupied by a flexible string that vibrates under the pressure of the fluid on the plane where $\Omega$ lies. The displacement of $\Gamma_{0}$, described by the scalar function $w=w(x, t)$, obeys the one-dimensional dissipative wave equation. As $\Omega$ is compressible fluid where the velocity field $v$ is given by the potential $\varphi=\varphi(x, y, t),(v=\nabla \varphi)$. All deformations are supposed to be small enough so that linear theory applies.

The linear motion of this system is described by means of the coupled wave equations

$$
\begin{cases}\rho \varphi_{t t}-c \Delta \varphi+\gamma_{1} \varphi_{t}=0 & \text { in } \Omega \times(0, \infty) \\ \frac{\partial \varphi}{\partial v}=0 & \text { on } \Gamma_{1} \times(0, \infty) \\ \frac{\partial \varphi}{\partial v}=-w_{t} & \text { on } \Gamma_{0} \times(0, \infty) \\ \varphi=0 & \text { on } \Gamma_{2} \times(0, \infty) \\ w_{t t}-w_{x x}+\gamma_{0} w_{t}+c \varphi_{t}=0 & \text { on } \Gamma_{0} \times(0, \infty) \\ w_{x}(0, t)=w_{x}(1, t)=0 & \text { for } t>0 \\ \varphi(0)=\varphi^{0}, \quad \varphi_{t}(0)=\varphi^{1} & \text { in } \Omega \\ w(0)=w^{0}, \quad w_{t}(0)=w^{1} & \text { on } \Gamma_{0}\end{cases}
$$

where $v$ denote the unit outward normal to $\Omega$.

We define the energy associated with this system. Proceeding formally, multiply the first equation by $\varphi_{t}$ and then integrate over $\Omega$.

$$
\frac{1}{2} \frac{\mathrm{d}}{\mathrm{d} t} \int_{\Omega}\left|\varphi_{t}\right|^{2} \mathrm{~d} x \mathrm{~d} y+\frac{c}{\rho} \int_{\Omega} \nabla \varphi \cdot \nabla \varphi_{t} \mathrm{~d} x \mathrm{~d} y-\frac{c}{\rho} \int_{\Gamma} \frac{\partial \varphi}{\partial v} \cdot \varphi_{t} \mathrm{~d} \sigma+\frac{\gamma_{1}}{\rho} \int_{\Omega}\left|\varphi_{t}\right|^{2} \mathrm{~d} x \mathrm{~d} y=0 .
$$

However, the integral

$$
\int_{\Gamma} \frac{\partial \varphi}{\partial v} \cdot \varphi_{t} \mathrm{~d} \sigma=\int_{\Gamma_{1}} \underbrace{\frac{\partial \varphi}{\partial v}}_{=0} \cdot \varphi_{t} \mathrm{~d} \sigma+\int_{\Gamma_{0}} \underbrace{\frac{\partial \varphi}{\partial v}}_{-w_{t}} \cdot \varphi_{t} \mathrm{~d} \sigma-\int_{\Gamma_{2}} \frac{\partial \varphi}{\partial v} \cdot \underbrace{\varphi_{t}}_{=0} \sigma,
$$

which leads us

$$
\int_{\Gamma} \frac{\partial \varphi}{\partial v} \cdot \varphi_{t} \mathrm{~d} \sigma=\int_{\Gamma_{0}} \frac{\partial \varphi}{\partial v} \cdot \varphi_{t}=-\int_{\Gamma_{0}} w_{t} \varphi_{t} \mathrm{~d} x
$$

Replacing (3) into (2) we obtain

$$
\frac{\mathrm{d}}{\mathrm{d} t}\left[\frac{1}{2} \int_{\Omega}\left|\varphi_{t}\right|^{2} \mathrm{~d} x \mathrm{~d} y+\frac{c}{\rho}|\nabla \varphi|^{2}\right]+\frac{\gamma_{1}}{\rho} \int_{\Omega}\left|\varphi_{t}\right|^{2} \mathrm{~d} x \mathrm{~d} y-\frac{c}{\rho} \int_{\Gamma_{0}} w_{t} \varphi_{t} \mathrm{~d} x=0 .
$$

Multiplying by $w$ in the second equation of the system (1) and then integrate over $\Gamma_{0}$

$$
\frac{1}{2 \rho} \frac{\mathrm{d}}{\mathrm{d} t} \int_{\Gamma_{0}}\left|w_{t}\right|^{2} \mathrm{~d} x-\frac{1}{\rho} \int_{\Gamma_{0}} w_{x x} w_{t} \mathrm{~d} x+\frac{\gamma_{0}}{\rho} \int_{\Gamma_{0}}\left|w_{t}\right|^{2} \mathrm{~d} x+\frac{c}{\rho} \int_{\Gamma_{0}} \varphi_{t} w_{t} \mathrm{~d} x=0 .
$$


Integrating by parts

$$
\int_{\Gamma_{0}} w_{x x} w_{t} \mathrm{~d} x=\left.\underbrace{w_{x}} w_{t}\right|_{0} ^{1}-\int_{\Gamma_{0}} w_{x} w_{t x} \mathrm{~d} x=-\frac{1}{2} \frac{\mathrm{d}}{\mathrm{d} t} \int_{\Gamma_{0}}\left|w_{x}\right|^{2} \mathrm{~d} x .
$$

Replacing the above equation over (5) we obtain

$$
\frac{1}{2 \rho} \frac{\mathrm{d}}{\mathrm{d} t} \int_{\Gamma_{0}}\left|w_{t}\right|^{2} \mathrm{~d} x+\frac{1}{2 \rho} \frac{\mathrm{d}}{\mathrm{d} t} \int_{\Gamma_{0}}\left|w_{x}\right|^{2} \mathrm{~d} x+\frac{\gamma_{0}}{\rho} \int_{\Gamma_{0}}\left|w_{t}\right|^{2} \mathrm{~d} x+\frac{c}{\rho} \int_{\Gamma_{0}} \varphi_{t} w_{t} \mathrm{~d} x=0,
$$

which leads us to assert that, the energy of the system is given by

$$
E(t)=E(\varphi, w ; t)=\frac{1}{2} \int_{\Omega}\left|\varphi_{t}\right|^{2}+\frac{c}{\rho}|\nabla \varphi|^{2} \mathrm{~d} x \mathrm{~d} y+\frac{1}{2 \rho} \int_{\Gamma_{0}} w_{x}^{2}+w_{t}^{2} \mathrm{~d} x,
$$

for each $t \geq 0$.

Remark 1 The first two terms represents the energy of acoustic wave and the other terms is the energy of bungee wave.

The system has a natural dissipation. Indeed, to observe this fact multiply the first equation of (1) by $\varphi_{t}$ and then the second equation of (1) by $w_{t}$, as was done in previous calculations

$$
\frac{\mathrm{d} E(t)}{\mathrm{d} t}=-\frac{\gamma_{1}}{\rho} \int_{\Omega}\left|\varphi_{t}\right|^{2} \mathrm{~d} x \mathrm{~d} y-\frac{\gamma_{0}}{\rho} \int_{\Gamma_{0}} w_{t}^{2} \mathrm{~d} x<0,
$$

if $\gamma_{1}^{2}+\gamma_{0}^{2} \neq 0$. Micu, S. in his doctoral thesis [7] shows non-exponential decay of the energy of the hybrid system (1).

\section{Mathematical Formulation}

Define the face space $\mathcal{H}=H^{1}(\Omega) \times L^{2}(\Omega) \times H^{1}\left(\Gamma_{0}\right) \times L^{2}\left(\Gamma_{0}\right)$ endowed with the Hilbertian scalar product given by

$$
\langle U, V\rangle=\int_{\Omega} \frac{c}{\rho} \nabla \varphi_{1} \cdot \nabla \psi_{1}+\varphi_{2} \psi_{2} \mathrm{~d} x \mathrm{~d} y+\frac{1}{\rho} \int_{\Gamma_{0}}\left(\varphi_{3}\right)_{x}\left(\psi_{3}\right)_{x}+\varphi_{4} \psi_{4} \mathrm{~d} x,
$$

for all $U=\left(\varphi_{1}, \varphi_{2}, \varphi_{3}, \varphi_{4}\right), V=\left(\psi_{1}, \psi_{2}, \psi_{3}, \psi_{4}\right) \in \mathcal{H}$. We can show that the pair $(\mathcal{H},\langle\rangle$,$) is a Hilbert space.$

Since the first and second equation of the system (1), we obtain

$$
\begin{gathered}
\varphi_{t}=\varphi_{2} \text { then } \varphi_{2 t}=\frac{c}{\rho} \Delta \varphi_{1}-\frac{\gamma_{1}}{\rho} \varphi_{2} \\
w_{t}=\varphi_{4} \text { then } \varphi_{4 t}=\left(\varphi_{3}\right)_{x x}-\gamma_{0} \varphi_{4}-c \varphi_{2} .
\end{gathered}
$$

These equations lead us to define the operator $\mathcal{A}: \mathcal{H} \rightarrow \mathcal{H}$ by

$$
\mathcal{A}:=\left[\begin{array}{cccc}
0 & 1 & 0 & 0 \\
\frac{c}{\rho} \Delta & -\frac{\gamma_{1}}{\rho} & 0 & 0 \\
0 & 0 & 0 & 1 \\
0 & -c & (\cdot)_{x x} & -\gamma_{0}
\end{array}\right],
$$

in this sense for all $U=\left(\varphi_{1}, \varphi_{2}, \varphi_{3}, \varphi_{4}\right) \in \mathcal{H}$,

$$
\mathcal{A} U=\left(\varphi_{2}, \frac{c}{\rho} \Delta \varphi_{1}-\frac{\gamma_{1}}{\rho} \varphi_{2}, \varphi_{4},\left(\varphi_{3}\right)_{x x}-\gamma_{0} \varphi_{4}-c \varphi_{2}\right) .
$$

Note that $\mathcal{A} U \in \mathcal{H}$ if and only if 


$$
\begin{aligned}
& \varphi_{2} \in H^{1}(\Omega), \quad \frac{c}{\rho} \Delta \varphi_{1}-\frac{\gamma_{1}}{\rho} \varphi_{2} \in L^{2}(\Omega), \quad \frac{c}{\rho} \Delta \varphi_{1}-\frac{\gamma_{1}}{\rho} \varphi_{2}, \quad \varphi_{4} \in H^{1}\left(\Gamma_{0}\right) \\
& -c \varphi_{2}+\left(\varphi_{3}\right)_{x x}-\gamma_{0} \varphi_{4} \in L^{2}\left(\Gamma_{0}\right) .
\end{aligned}
$$

Now, we consider the problem with Neumann boundary conditions

$$
\left\{\begin{array}{l}
\frac{c}{\rho} \Delta \varphi_{1}=\frac{\gamma_{1}}{\rho} \varphi_{2}+h \in L^{2}(\Omega), \quad h \in L^{2}(\Omega) \\
\frac{\partial}{\partial v} \varphi_{1}=0 \quad \text { on } \Gamma_{1} \\
\frac{\partial}{\partial v} \varphi_{1}=\varphi_{4} \quad \text { on } \Gamma_{0} \\
\varphi_{1}=0 \quad \text { on } \Gamma_{2},
\end{array}\right.
$$

where we can say that $\varphi_{1} \in H^{2}(\Omega)$ see [9]. Similarly, consider the problem

$$
\left\{\begin{array}{l}
\left(\varphi_{2}\right)_{x x}=c \varphi_{2}+\gamma_{0} \varphi_{4} \in L^{2}\left(\Gamma_{0}\right) \\
\left(\varphi_{3}\right)_{x}(0)=\left(\varphi_{3}\right)_{x}(1)=0 .
\end{array}\right.
$$

We can say that $\varphi_{2} \in H^{2}\left(\Gamma_{0}\right)$. In this sense we can define the domain of the operator $\mathcal{A}$ which we denote $\mathcal{D}(\mathcal{A})$, as the set of $U=\left(\varphi_{1}, \varphi_{2}, \varphi_{3}, \varphi_{4}\right) \in \mathcal{H}$ such that $\varphi_{1} \in H^{2}(\Omega), \quad \varphi_{2} \in H^{1}(\Omega), \quad \varphi_{3} \in H^{2}\left(\Gamma_{0}\right)$, $\varphi_{4} \in H^{1}\left(\Gamma_{0}\right)$ satisfying

$$
\begin{aligned}
& \frac{\partial \varphi_{1}}{\partial v}=0 \quad \text { on } \Gamma_{1} \\
& \frac{\partial \varphi_{1}}{\partial v}=\varphi_{4} \quad \text { on } \Gamma_{0} \\
& \varphi_{1}=0 \quad \text { on } \Gamma_{2} \\
& \left(\varphi_{3}\right)_{x}(0)=\left(\varphi_{3}\right)_{x}(1)=0 .
\end{aligned}
$$

Remark 2 By previous observations we can say that the hybrid system (1) is equivalent to the Cauchy problem

$$
\left\{\begin{array}{l}
U_{t}(t)=\mathcal{A} U(t), t>0 \\
U(0)=U_{0},
\end{array}\right.
$$

where $U_{0}=\left(\varphi^{0}, \varphi^{1}, \omega^{0}, \omega^{1}\right) \in \mathcal{D}(\mathcal{A})$ and $U(t)=\left(\varphi_{1}, \varphi_{2}, \varphi_{3}, \varphi_{4}\right) \in \mathcal{D}(\mathcal{A}), t \geq 0$.

\section{Solution Existence}

We want to show that $\mathcal{A}$ is a dissipative operator and $0 \in \rho(\mathcal{A})$ (The resolvent set of $\mathcal{A}$ ).

Remark 3 The operator $\mathcal{A}$ is dissipative, ie $\langle\mathcal{A} U, U\rangle<0$ for all $U=\left(\varphi_{1}, \varphi_{2}, \varphi_{3}, \varphi_{4}\right) \in \mathcal{D}(\mathcal{A})$.

Applying (9), we get

$$
\langle\mathcal{A} U, U\rangle=-\frac{\gamma_{1}}{\rho} \int_{\Omega} \varphi_{2}^{2} \mathrm{~d} x \mathrm{~d} y-\frac{\gamma_{0}}{\rho} \int_{\Gamma_{0}} \varphi_{4}^{2} \mathrm{~d} x<0
$$

\section{Resolvent Equation:}

Given $F \in \mathcal{H}$, we find $U \in \mathcal{D}(\mathcal{A})$

$$
(\lambda I-\mathcal{A}) U=F, \quad \lambda \in \mathbb{C} .
$$

In particular, $0 \in \rho(\mathcal{A})$, if and only if, there is 
P. Gambia et al.

$$
U \in \mathcal{D}(\mathcal{A}) ; \quad-\mathcal{A} U=F
$$

that is,

$$
\begin{gathered}
-\varphi_{2}=F_{1} \\
-\frac{c}{\rho} \Delta \varphi_{1}+\frac{\gamma_{1}}{\rho} \varphi_{2}=F_{2} \\
-\varphi_{4}=F_{3} \\
-\left(\varphi_{3}\right)_{x x}+\gamma_{0} \varphi_{4}+c \varphi_{2}=F_{4},
\end{gathered}
$$

where $F=\left(F_{1}, F_{2}, F_{3}, F_{4}\right)$. By previous observations that there have $U \in \mathcal{D}(\mathcal{A})$. Using the application of bummer Phillips Theorem [10] [11], we have the following result.

Theorem 1 The operator $\mathcal{A}$ set to (10) is the infinitesimal generator of a contraction semigroup $\mathrm{C}_{0}$.

Theorem 2 The $\mathcal{A}$ is the infinitesimal generator of a semigroup $\mathrm{C}_{0}$ and verifies $U_{0} \in \mathcal{D}\left(\mathcal{A}^{3}\right)$ then the solution of (13) satisfies

$$
U \in C^{1}(0, \infty ; \mathcal{H}) \cap C^{2}(0, \infty ; \mathcal{D}(\mathcal{A}))
$$

4. Asymptotic Behavior

We now show that the energy associated with the system decays exponentially. Multiplying by $\varphi$ the first eqcation in (1) and integrating over $\Omega$ yields

$$
\int_{\Omega} \varphi_{t t} \varphi \mathrm{d} x \mathrm{~d} y-\frac{c}{\rho} \int_{\Omega} \Delta \varphi \varphi \mathrm{d} x \mathrm{~d} y+\frac{\gamma_{1}}{\rho} \int_{\Omega} \varphi_{t} \varphi \mathrm{d} x \mathrm{~d} y=0,
$$

equivalently

$$
\frac{\mathrm{d}}{\mathrm{d} t} \int_{\Omega} \varphi_{t} \varphi \mathrm{d} x \mathrm{~d} y-\int_{\Omega}\left|\varphi_{t}\right|^{2} \mathrm{~d} x \mathrm{~d} y+\frac{c}{\rho} \int_{\Omega}|\nabla \varphi|^{2} \mathrm{~d} x \mathrm{~d} y-\frac{c}{\rho} \int_{\Gamma_{0}} \frac{\partial \varphi}{\partial v} \varphi \sigma+\frac{\gamma_{1}}{\rho} \int_{\Omega} \varphi_{t} \varphi \mathrm{d} x \mathrm{~d} y=0 .
$$

Observe that

$$
-\frac{c}{\rho} \int_{\Gamma_{0}} \frac{\partial \varphi}{\partial v} \varphi \sigma=\frac{c}{\rho} \frac{\mathrm{d}}{\mathrm{d} t} \int_{\Gamma_{0}} \omega \varphi \mathrm{d} x-\frac{c}{\rho} \int_{\Gamma_{0}} \omega \varphi_{t} \mathrm{~d} x
$$

From the second equation in (1), we obtain

$$
-\frac{c}{\rho} \int_{\Gamma_{0}} \omega \varphi_{t} \mathrm{~d} x=-\frac{1}{\rho} \int_{\Gamma_{0}} \omega\left(-\omega_{t t}+\omega_{x x}-\gamma_{0} \omega\right) \mathrm{d} x
$$

On the other hand,

$$
\frac{1}{\rho} \int_{\Gamma_{0}} \omega \omega_{t t} \mathrm{~d} x=\frac{1}{\rho} \frac{\mathrm{d}}{\mathrm{d} t} \int_{\Gamma_{0}} \omega \omega_{t} \mathrm{~d} x-\frac{1}{\rho} \int_{\Gamma_{0}} \omega_{t}^{2} \mathrm{~d} x
$$

From (17)-(19), we obtain

$$
-\frac{c}{\rho} \int_{\Gamma_{0}} \frac{\partial \varphi}{\partial v} \varphi \sigma=\frac{\mathrm{d}}{\mathrm{d} t}\left[\frac{1}{\rho} \int_{\Gamma_{0}} \omega \omega_{t} \mathrm{~d} x+\frac{c}{\rho} \int_{\Gamma_{0}} \omega \varphi \mathrm{d} x\right]+\frac{1}{\rho} \int_{\Gamma_{0}} \omega_{x}^{2} \mathrm{~d} x-\frac{1}{\rho} \int_{\Gamma_{0}} \omega_{t}^{2} \mathrm{~d} x+\frac{\gamma_{0}}{\rho} \int_{\Gamma_{0}} \omega \omega_{t} \mathrm{~d} x .
$$

Replacing (20) into (16)

$$
\begin{aligned}
& \frac{\mathrm{d}}{\mathrm{d} t} \int_{\Omega} \varphi_{t} \varphi \mathrm{d} x \mathrm{~d} y-\int_{\Omega}\left|\varphi_{t}\right|^{2} \mathrm{~d} x \mathrm{~d} y+\frac{c}{\rho} \int_{\Omega}|\nabla \varphi|^{2} \mathrm{~d} x \mathrm{~d} y+\frac{\mathrm{d}}{\mathrm{d} t}\left[\frac{1}{\rho} \int_{\Gamma_{0}} \omega \omega_{t} \mathrm{~d} x+\frac{c}{\rho} \int_{\Gamma_{0}} \omega \varphi \mathrm{d} x\right] \\
& +\frac{1}{\rho} \int_{\Gamma_{0}} \omega_{x}^{2} \mathrm{~d} x-\frac{1}{\rho} \int_{\Gamma_{0}} \omega_{t}^{2} \mathrm{~d} x+\frac{\gamma_{0}}{\rho} \int_{\Gamma_{0}} \omega \omega_{t} \mathrm{~d} x+\frac{\gamma_{1}}{\rho} \int_{\Omega} \varphi_{t} \varphi \mathrm{d} x \mathrm{~d} y=0,
\end{aligned}
$$

1232 
P. Gamboa et al.

or equivalently

$$
\begin{aligned}
& \frac{\mathrm{d}}{\mathrm{d} t}\left[\int_{\Omega} \varphi_{t} \varphi \mathrm{d} x \mathrm{~d} y+\frac{1}{\rho} \int_{\Gamma_{0}} \omega \omega_{t} \mathrm{~d} x+\frac{c}{\rho} \int_{\Gamma_{0}} \omega \varphi \mathrm{d} x\right]-\int_{\Omega}\left|\varphi_{t}\right|^{2} \mathrm{~d} x \mathrm{~d} y+\frac{c}{\rho} \int_{\Omega}|\nabla \varphi|^{2} \mathrm{~d} x \mathrm{~d} y \\
& +\frac{1}{\rho} \int_{\Gamma_{0}} \omega_{x}^{2} \mathrm{~d} x-\frac{1}{\rho} \int_{\Gamma_{0}} \omega_{t}^{2} \mathrm{~d} x+\frac{\gamma_{0}}{\rho} \int_{\Gamma_{0}} \omega \omega_{t} \mathrm{~d} x+\frac{\gamma_{1}}{\rho} \int_{\Omega} \varphi_{t} \varphi \mathrm{d} x \mathrm{~d} y=0 .
\end{aligned}
$$

Now, since Poincare inequality we have

$$
\left|\frac{\gamma_{0}}{\rho} \int_{\Gamma_{0}} \omega \omega_{t}\right| \leq \frac{\gamma_{0} \lambda_{1}}{\rho}\left[\int_{\Gamma_{0}} \omega_{x}^{2}\right]^{1 / 2}\left[\int_{\Gamma_{0}} \omega_{t}^{2} \mathrm{~d} x\right]^{1 / 2} \leq \frac{\alpha}{2 \rho} \int_{\Gamma_{0}} \omega_{x}^{2} \mathrm{~d} x+\frac{\gamma_{0} \lambda_{1}}{2 \alpha} \int_{\Gamma_{0}} \omega_{t}^{2},
$$

where $\lambda_{1}$ is the Poincare constant. In a similar way,

$$
\left|\frac{\gamma_{1}}{\rho} \int_{\Omega} \varphi \varphi_{t} \mathrm{~d} x \mathrm{~d} y\right| \leq \frac{\theta}{2 \rho} \int_{\Omega}|\nabla \varphi|^{2} \mathrm{~d} x \mathrm{~d} y+\frac{\gamma_{1} \lambda_{1}}{2 \theta \rho} \int_{\Omega}\left|\varphi_{t}\right|^{2} \mathrm{~d} x \mathrm{~d} y .
$$

From (22), (23) and (24) we have

$$
\begin{aligned}
& \frac{\mathrm{d}}{\mathrm{d} t}\left[\int_{\Omega} \varphi_{t} \varphi \mathrm{d} x \mathrm{~d} y+\frac{1}{\rho} \int_{\Gamma_{0}} \omega \omega_{t} \mathrm{~d} x+\frac{c}{\rho} \int_{\Gamma_{0}} \omega \varphi \mathrm{d} x\right] \\
& \leq\left(1+\frac{\gamma_{1} \lambda_{1}}{2 c \rho}\right) \int_{\Omega}\left|\varphi_{t}\right|^{2} \mathrm{~d} x \mathrm{~d} y-\frac{3 c}{4 \rho} \int_{\Omega}|\nabla \varphi|^{2} \mathrm{~d} x \mathrm{~d} y+\frac{1}{\rho} \int_{\Gamma_{0}} \omega_{t}^{2} \mathrm{~d} x-\frac{1}{2} \int_{\Gamma_{0}} \omega_{x}^{2} \mathrm{~d} x .
\end{aligned}
$$

We define the operator

$$
\mathcal{L}(t):=n E(t)+\int_{\Omega} \varphi_{t} \varphi \mathrm{d} x \mathrm{~d} y+\frac{1}{\rho} \int_{\Gamma_{0}} \omega \omega_{t} \mathrm{~d} x+\frac{c}{\rho} \int_{\Gamma_{0}} \omega \varphi \mathrm{d} x, \quad n \in \mathbb{N} .
$$

Differentiating (26) and using (8) we obtain

$$
\begin{aligned}
\frac{\mathrm{d}}{\mathrm{d} t} \mathcal{L}(t)= & n \frac{\mathrm{d}}{\mathrm{d} t} E(t)+\frac{\mathrm{d}}{\mathrm{d} t}\left[\int_{\Omega} \varphi_{t} \varphi \mathrm{d} x \mathrm{~d} y+\frac{1}{\rho} \int_{\Gamma_{0}} \omega \omega_{t} \mathrm{~d} x+\frac{c}{\rho} \int_{\Gamma_{0}} \omega \varphi \mathrm{d} x\right] \\
\leq & {\left[n \frac{\gamma_{1}}{\rho}-\left(1+\frac{\gamma_{1} \lambda_{1}}{2 c \rho}\right)\right] \int_{\Omega}\left|\varphi_{t}\right|^{2} \mathrm{~d} x \mathrm{~d} y-\frac{3 c}{4 \rho} \int_{\Omega}|\nabla \varphi|^{2} \mathrm{~d} x \mathrm{~d} y } \\
& -\left[n \frac{\gamma_{0}}{\rho}-\left(1+\frac{\gamma_{0} \lambda_{1}}{2 \rho}\right)\right] \int_{\Gamma_{0}} \omega_{t}^{2} \mathrm{~d} x-\frac{1}{2} \int_{\Gamma_{0}} \omega_{x}^{2},
\end{aligned}
$$

Considering $n$ large enough, we can obtain a constant $C$ such that

$$
\frac{\mathrm{d}}{\mathrm{d} t} \mathcal{L}(t) \leq-C E(t)
$$

On the other hand, using Poincaré, we can obtain

$$
\left|\int_{\Omega} \varphi \varphi_{t} \mathrm{~d} x \mathrm{~d} y\right| \leq \frac{c_{0} \delta \lambda_{1}}{2} \int_{\Omega}|\nabla \varphi|^{2} \mathrm{~d} x \mathrm{~d} y+\frac{1}{2 \delta} \int_{\Omega}\left|\varphi_{t}\right|^{2} \mathrm{~d} x \mathrm{~d} y .
$$

In a similar way

$$
\left|\frac{c}{\rho} \int_{\Gamma_{0}} \omega \varphi \mathrm{d} x\right| \leq \frac{c \lambda_{1}}{\rho}\left(\int_{\Gamma_{0}} \omega_{x}^{2}\right)^{1 / 2}\left(\int_{\Gamma_{0}}|\varphi|^{2} \mathrm{~d} x\right)^{1 / 2} \leq \frac{c \lambda_{1}}{2 \rho}\left(\theta \int_{\Gamma_{0}} \omega_{x}^{2} \mathrm{~d} x+\frac{1}{\theta} \int_{\Gamma_{0}}|\varphi|^{2} \mathrm{~d} x\right)
$$

Moreover, from trace

$$
\int_{\Gamma_{0}} \varphi^{2} \mathrm{~d} x \leq \int_{\Gamma} \varphi^{2} \mathrm{~d} x \leq c_{3} \int_{\Omega}|\nabla \varphi|^{2} \mathrm{~d} x \mathrm{~d} y .
$$

1233 
Replacing (31) into (30) we have

$$
\left|\frac{c}{\rho} \int_{\Gamma_{0}} \omega \varphi\right| \leq \frac{c \lambda_{1} \theta}{2 \rho} \int_{\Gamma_{0}} \omega_{x}^{2} \mathrm{~d} x+\frac{c \lambda_{1} c_{3}}{2 \rho \theta} \int_{\Omega}|\nabla \varphi|^{2} \mathrm{~d} x \mathrm{~d} y .
$$

From (23), (29), (32) and (26) we can to claim that there is a constant $\kappa_{0}$ and $\kappa_{1}$ such that

$$
\kappa_{0} E(t) \leq \mathcal{L}(t) \leq \kappa_{1} E(t)
$$

leading to decay exponentially energy

$$
E(t) \leq c_{2} E(0) \mathrm{e}^{-\kappa t}, \quad \forall t>0 .
$$

where $\kappa=C \kappa_{0}$. The result follows.

Remark 4 In the case of $\gamma_{1}=0$ can be also said that a power decays exponentially.

The above results support the conclusion.

Theorem 3 If $\left(\varphi_{0}, \varphi_{1}, \omega_{0}, \omega_{1}\right) \in \mathcal{D}(\mathcal{A})$ and $\gamma_{0} \neq 0$ then the solution $(\varphi, \omega)$ of the hybrid system (1) decays exponentially over time.

\section{Acknowledgements}

Octavio Vera thanks the support of the Fondecyt project 1121120.

\section{References}

[1] Banks, H.T., Fang, W., Silcox, R.J. and Smith, R.C. (1993) Aproximation Methods for Control of Acustic/Structures Models with Piezoceramic Actuators. Journal of Intelligent Material Systems and Structures, 4, 98-116. http://dx.doi.org/10.1177/1045389X9300400113

[2] Micu, S. and Zuazua, E. (1996) Stabilization and Periodic Solutions of a Hybrid System Arising in the Control of Noise. Proceedings of the IFIP TC7/WG-7.2 International Conference, Laredo, Lecture Notes in Pure and Applied Mathematics, Vol. 174, Marcel Dekker, New York, 219-230.

[3] Micu, S. (1999) Periodic Solutions for a Bidimensional Hybrid System Arising in the Control of Noise. Advances in Differential Equations, 4, 529-560.

[4] Cindea, N., Micu, S. and Pazoto, A.F. (2012) Periodic Solutions for a Weakly Dissipated Hybrid System. Journal of Mathematical Analysis and Applications, 385, 399-413. http://dx.doi.org/10.1016/j.jmaa.2011.06.049

[5] Hansen, S. and Zuazua, E. (1995) Exact Controllability and Stabilization of a Vibrating String with on Interior Point Mass. SIAM Journal on Control and Optimization, 33, 1357-1391. http://dx.doi.org/10.1137/S0363012993248347

[6] Littman, W. and Marcus, L. (1988) Exact Boundary Controllability of a Hybrid System of Elasticity. Archive for Rational Mechanics and Analysis, 103, 193-236. http://dx.doi.org/10.1007/BF00251758

[7] Micu, S.D. (1995) Analisis de un Sistema Hibrido Bidimensional Fluido-Estructura. Ph.D. Thesis, Universidad Complutense de Madrid, Madrid.

[8] Avalos, G. and Lasiecka, I. (2003) Exact Controllability of Structural Acoustic Interactions. Journal de Mathématiques Pures et Appliquées, 82, 1047-1073.

[9] Grisvard, P. (1985) Elliptic Problems in Non-Smooth Domains. Pitman.

[10] Muñoz Rivera, J.E. (2007) Semigrupos e Equações Diferenciais Parciais. Textos Pós Graduação, LNCC.

[11] Pazy, A. (1983) Semi-Groups of Linear Operators and Applications to Partial Differential Equations. Springer-Verlag. http://dx.doi.org/10.1007/978-1-4612-5561-1 